\title{
Imipenem in burn patients: pharmacokinetic profile and PK/PD target attainment
}

\author{
David S Gomez ${ }^{1}$, Cristina Sanches-Giraud ${ }^{2}$, Carlindo V Silva $\mathrm{Jr}^{3}$, Amanda M Ribas Rosa Oliveira ${ }^{1}$, \\ Joao Manoel da Silva Jr${ }^{1}$, Rolf Gemperli ${ }^{1}$ and Silvia RCJ Santos ${ }^{3}$
}

Unpredictable pharmacokinetics (PK) in burn patients may result in plasma concentrations below concentrations that are effective against common pathogens. The present study evaluated the imipenem PK profile and pharmacokinetic/ pharmacodynamics (PK/PD) correlation in burn patients. Fifty-one burn patients, 38.7 years of age (mean), $68.0 \mathrm{~kg}, 36.3 \%$ total burn surface area (TBSA), of whom $84 \%(43 / 51)$ exhibited thermal injury, $63 \%$ inhalation injury and $16 \%$ electrical injury (8/51), all of whom were receiving imipenem treatment were investigated. Drug plasma monitoring, PK study (120 sets of plasma levels) and PK/PD correlation were performed in a series of blood samples. Only $250 \mu$ l of plasma samples were required for drug plasma measurements using the ultra filtration technique for the purification of biological matrix and quantification using liquid chromatography. Probability of target attainment (PTA) was calculated using a PD target of $40 \%$ free drug concentrations above the minimum inhibitory concentration (40\%fT > MIC). Significant differences in PK parameters (medians), such as biological half-life $(2.2$ vs $5.5 \mathrm{~h})$, plasma clearance $(16.2$ vs $1.4 \mathrm{lh}-1)$ and volume of distribution $(0.86$ vs $0.19 \mathrm{l} \mathrm{kg}-1)$, were registered in burn patients via comparisons of set periods with normal renal function against periods of renal failure. Correlations between creatinine clearance and total body plasma clearance were also obtained. In addition, the PK profile did not change according to TBSA during sets when renal function was preserved. PTA was $>89 \%$ for MIC values up to $4 \mathrm{mg} \mathrm{I}^{-1}$. In conclusion, imipenem efficacy for the control of hospital infection on the basis of PK/PD correlation was guaranteed for burn in patients at the recommended dose regimens for normal renal function $\left(31.1 \pm 9.7 \mathrm{mg} \mathrm{kg}^{-1}\right.$ daily), but the daily dose must be reduced to $17.2 \pm 9.7 \mathrm{mg} \mathrm{kg}^{-1}$ during renal failure to avoid neurotoxicity.

The Journal of Antibiotics (2015) 68, 143-147; doi:10.1038/ja.2014.121; published online 17 September 2014

\section{INTRODUCTION}

A large number of factors may affect the pharmacokinetics (PK) of drugs in burn patients, such as total burn surface area and depth, sepsis, degree of hydration, serum protein concentrations, age, creatinine clearance and time after injury, which leads to changes in antimicrobial plasma concentrations, and consequently, antimicrobial killing activity could be altered. The disruption of the normal skin barrier and the immunocompromised state makes this population an easy target for colonization. ${ }^{1-3}$ Consequently, a higher dose appears necessary even when the pathogens have minimum inhibitory concentration (MIC) values typical of susceptible microorganisms. ${ }^{4}$

Therapeutic drug monitoring is strongly recommended to optimize drug exposure in individual patients. In addition, the pharmacokinetic-pharmacodynamic (PK/PD) relationship is the major determinant of drug efficacy for antimicrobial agents, and this relationship allows dose regimen optimization to improve patient outcome and reduce the selection of resistant mutants. ${ }^{4}$

Imipenem, a carbapenem derivative, exhibits similar kill characteristics to other beta-lactams, but this carbapenem exhibits some post-antimicrobial effects. The literature on the influence of burn injury on imipenem $\mathrm{PK}$, primarily plasma clearance and the volume of distribution, is conflicting. ${ }^{3,5-7}$

Therefore, an improved understanding of the PK/PD characteristics of imipenem in the burn population is extremely important to maximize its bactericidal activity and minimize the risk of selecting pathogens. The present study described the pharmacokinetic profile of imipenem at steady-state levels in burn patients and investigated target attainment related to in vivo data (that is, drug plasma concentration/ PK parameters) and in vitro data (MIC) by applying the PK/PD correlation.

\section{MATERIALS AND METHODS}

Study design and patients eligibility

This study was a prospective, open-label study, approved by the institutional ethical committee of the hospital. Written informed consent was obtained from all patients or their legally designated representatives before study entry. Patients over 18 years old from the Intensive Care Burn Unit (ICBU) presenting severe thermal injury and clinical and laboratory diagnosis of sepsis

\footnotetext{
${ }^{1}$ Plastic Surgery and Burns, Hospital das Clinicas, Medical School, University of Sao Paulo, Sao Paulo, Brazil; ${ }^{2}$ Department of Pharmacy, Universidade Federal de São João del Rei, Divinópolis, Brazil and ${ }^{3}$ School of Pharmaceutical Sciences University of Sao Paulo, Sao Paulo, Brazil

Correspondence: Professor SRCJ Santos, Clinical Pharmacology \& Therapeutics, Department of Pharmacy, School of Pharmaceutical Sciences, University of Sao Paulo, Rua Perucaia, 63, Sao Paulo, SP 05578-070, Brazil.

E-mail: pharther@usp.br

Received 13 February 2014; revised 9 May 2014; accepted 25 July 2014; published online 17 September 2014
} 
were eligible for inclusion. Patients with a basis for imipenem treatment for suspected or documented nosocomial infection were included. Patients with significant renal impairment $\left(\mathrm{CLcr}<50 \mathrm{ml} \mathrm{min}^{-1}\right)$ and hemodialysis requirements were also included.

Participants received i.v. imipenem as a part of their medical care. The intensive care medical team made decisions regarding the initiation of antimicrobial therapy and subsequent changes in dose on the basis of clinical evaluations, image data diagnosis, laboratory data, drug plasma levels/PK profile and $\mathrm{PK} / \mathrm{PD}$ correlation. Imipenem administration was performed via drug infusion over $0.5 \mathrm{~h}$, complete medical histories, including physical examination and a review of clinical, laboratory and image data, were obtained for each enrolled patient. Creatinine clearance (CLcr) was calculated using the Cockcroft and Gault method and the calculated ideal body weight considering gender. ${ }^{8}$

\section{Blood sampling}

Blood samples were obtained as a part of routine therapeutic drug monitoring for medical care during each patient's stay in the intensive care unit. Treatment duration was determined on the basis of the patient's clinical status and results of microbiological susceptibility testing.

Blood samples (four or five samples per set of plasma levels) were obtained from each patient at steady state levels (that is, at least five half-lives of imipenem treatment) through a central catheter and placed into tubes containing sodium EDTA ( $2 \mathrm{ml}$ each). Blood sampling was planned on the drug-dosing interval. Collected blood samples were centrifuged at $1800 \mathrm{~g}\left(5^{\circ} \mathrm{C}\right)$. A volume equivalent to $1 \mathrm{ml}$ of plasma was added to the same volume of a MOPS solution containing a mixture of $0.5 \mathrm{M}$ MOPS, ethylene glycol, purified water, $(2: 1: 1, \mathrm{v} / \mathrm{v})$ and stored $\left(-80^{\circ} \mathrm{C}\right)$ until quantification by the drug plasma assay detailed in the bioanalytical method below.

\section{Bioanalytical method}

Plasma samples $(250 \mu \mathrm{l})$ were added to a mixture containing 0.5 M MOPS ([3morpholino] propane sulfonic acid, Ultrapure Bioreagent J.T. Baker), ethylene glycol, purified water $(2: 1: 1, \mathrm{v} / \mathrm{v})$ and an internal standard (IS; ceftriaxone ( $10 \mu \mathrm{g}$ per assay) followed by an ultra filtration procedure using Amicon Ultra $30 \mathrm{~K}$ cutoffs $(0.5$ centrifugal filter device, Millipore Corporation, Darmstadt, Germany) for protein removal.

Chromatographic analysis was performed on a LC10A Class VP (Shimadzu, Kyoto, Japan) using an LC18 Supelcosil $(250 \times 4.6 \mathrm{~mm} 5$ micra $)$ column. The mobile phase consisted of $0.01 \mathrm{M}, \mathrm{pH} 7.0$ phosphate buffer plus acetonitrile $(99: 1, \mathrm{v} / \mathrm{v})$ at a flow rate of $0.8 \mathrm{ml} \mathrm{min}^{-1}$. A UV detector was set at $300 \mathrm{~nm}$, and peaks were eluted at $5.7 \mathrm{~min}$ (IS) and $12.3 \mathrm{~min}$ (imipenem). The bioanalytical method was validated, and the data obtained showed good linearity $(0.25-$ $\left.100 \mu \mathrm{g} \mathrm{ml}^{-1} ; r^{2} 0.9974\right)$, including the LLOQ $\left(0.25 \mu \mathrm{g} \mathrm{ml}^{-1} \mathrm{RSD}: 7.5 \%\right)$, LLOD $\left(0.12 \mu \mathrm{g} \mathrm{ml}^{-1}\right.$ RSD: $\left.14.8 \%\right)$, absolute recovery (93.6\%), inter/intraday imprecision (7.3/5.9\%), inter/intraday accuracy $(1.5 \% / 1.7 \%$, bias) and robustness. Stability was investigated using the freeze-thaw cycle with good reproducibility. In addition, the long-term stability of imipenem in plasma samples was evaluated for 90 days after the storage of biological matrices in an ultralow freezer $\left(-80^{\circ} \mathrm{C}\right)$. The accuracy, expressed as systematic error, was $2.1 / 2.7 / 14.2 \%$ for the high/medium/low quality control drug plasma concentrations, respectively.

\section{Pharmacokinetic analysis and PK/PD correlations}

Plasma concentration-time data were analyzed using a one-compartment open model and non-compartmental data analysis in PK Solutions 2.0 software (Summit, Montrose, CO, USA), and parameters at steady state were obtained. The estimated parameters included the terminal elimination rate constant (kel), biological half-life $\left(\mathrm{t}_{1 / 2 \beta}\right)$, area under the plasma concentration-time dosing interval curve $\left(\mathrm{AUC}_{\tau}^{\mathrm{ss}} \tau\right)$, plasma clearance $\left(\mathrm{CL}_{\mathrm{T}}\right)$ and apparent volume of distribution $\left(\mathrm{Vd}^{\mathrm{ss}}\right)$.

Minimum inhibitory concentrations (MIC) of potential pathogens isolated from a patient's fluids or secretions were based on MIC data $\left(0.5-4.0 \mathrm{mg}^{-1}\right)$ obtained from the Hospital's Central Laboratory or MIC values reported by the European Committee on Antimicrobial Susceptibility Testing (EUCAST) database. ${ }^{9}$

The PK/PD goal for imipenem was the percentage of dosing time that drug plasma levels (free fraction) remained above MIC $(\% f T>M I C)$, which should be equivalent at $40 \% \mathrm{~T}>$ MIC. ${ }^{5,10}$ The probability of target attainment, which is related to the effective drug plasma concentration, PK parameters and MIC $\left(0.5-4.0 \mathrm{mg} \mathrm{l}^{-1}\right)$, was calculated as the percentage of patient data sets in which the recommended target of $40 \% \mathrm{f} \mathrm{T}>\mathrm{MIC}$ was reached.

\section{Statistical analysis}

Demographic and pharmacokinetic data were analyzed using GraphPad Prism, version 4.0 (GraphPad Software, Inc., Chicago, IL, USA). Pharmacokinetic parameters were compared using a two-tailed paired Student's $t$-test and oneway analysis of variance. The Kruskal-Wallis test was used for PK, and Tukey's post hoc test was used to compare daily dose. Total plasma clearance and the estimated CLcr were plotted via the application of the Spearman's rank correlation for nonparametric data. Statistical significance for all performed tests was defined as a $P$-value $<0.05$.

\section{RESULTS}

Fifty-one burn patients were included in the study protocol from December 2010 to January 2013. Data obtained from 120 sets of imipenem plasma levels during the follow-up period were distributed into two groups based on renal function. Patients with preserved renal function (FRN) and end-stage renal failure (RF) were also considered.

The demographic data of the investigated patients and the characteristics of burn injury are described in Table 1 . The period of time after injury in burn patients on a clinical course in the intensive care unit was $23.7 \pm 13.1$ days (mean/s.d., minimum of 5 days and maximum of 82 days) (Table 1 ).

Thirty-six patients presented normal renal function (NRF: 73 sets), and renal failure was registered in 15 patients (RF: 47 sets). Eleven of these 15 patients exhibited renal failure at the beginning of antimicrobial treatment. Imipenem pharmacokinetic data in burn patients were stratified across normal renal function and renal failure (Table 2). Nonparametric statistical tests indicated that the significance related to PK parameters was registered by the comparison of RF against NRF, expressed as medians/quartiles.

Table 1 Patients demographic data $(n=51)$

\begin{tabular}{lcrrr}
\hline & No. (\%) & Mean/s.d. (min-max values) & C5\% Cl \\
\hline Age (years) & & $38.7 \pm 15.7(18-90)$ & $34.4-43.0$ \\
Weight (kg) & & $68.0 \pm 9.9(40-90)$ & 36.6 \\
TBSA (\%) & & $23.7 \pm 13.1(5-82)$ & \\
Time of accident (days) & $32 \mathrm{M} / 19 \mathrm{~F}$ & & \\
Gender & $43 / 51(84 \%)$ & & \\
Thermal injury & $08 / 51(16 \%)$ & & \\
Electrical injury & $32 / 51(63 \%)$ & & \\
Inhalation injury & & & \\
\hline
\end{tabular}

Abbreviations: $\mathrm{Cl}$, confidence interval; $\mathrm{F}$, female; M, male; No., number of subjects; TBSA, total burn surface area. 
Table 2 Pharmacokinetics (PK) of imipenem—daily dose required for burn patients according to renal function

\begin{tabular}{|c|c|c|c|c|}
\hline PK data & Parameters & NRF ( $\mathrm{n}=73$ sets $)$ & $R F(\mathrm{n}=47$ sets $)$ & P-value a \\
\hline \multirow[t]{2}{*}{$t_{(1 / 2) \beta}(h)$} & Median (IQ 25-75\%) & $3.1(1.7-5.6)$ & $5.5(4.3-8.5)$ & \multirow[t]{2}{*}{$<0.0001$} \\
\hline & Mean/s.d. (CV\%) & $2.8 \pm 1.8(63 \%)$ & $6.4 \pm 3.2(50 \%)$ & \\
\hline $\mathrm{CL}_{\mathrm{T}}\left(\mathrm{I} \mathrm{h}^{-1}\right)$ & Mean/s.d. (CV\%) & $17.8 \pm 6.4(36 \%)$ & $1.7 \pm 1.1(62 \%)$ & $<0.0001$ \\
\hline \multirow[t]{2}{*}{$V d^{s s}\left(\mathrm{~kg}^{-1}\right)$} & Median (IQ 25-75\%) & $0.86(0.60-1.28)$ & $0.19(0.14-0.23)$ & \multirow[t]{2}{*}{$<0.0001$} \\
\hline & Mean/s.d. (CV\%) & $1.01 \pm 0.58(59 \%)$ & $0.19 \pm 0.06(34 \%)$ & \\
\hline
\end{tabular}

Abbreviations: $\mathrm{CL}_{T}$, total plasma clearance; $\mathrm{CV}$, variability; $\mathrm{NRF}$, normal renal function; $\mathrm{RF}$, renal failure; $\mathrm{t}_{(1 / 2) \beta}$, biological half-life; Vdss, apparent volume of distribution at steady state. aStatistics: Mann-Whitney—Student's $t$-test. Data expressed as quartiles (median/IQ 25-75\%) for pharmacokinetics; mean/s.d. for daily dose and trough.

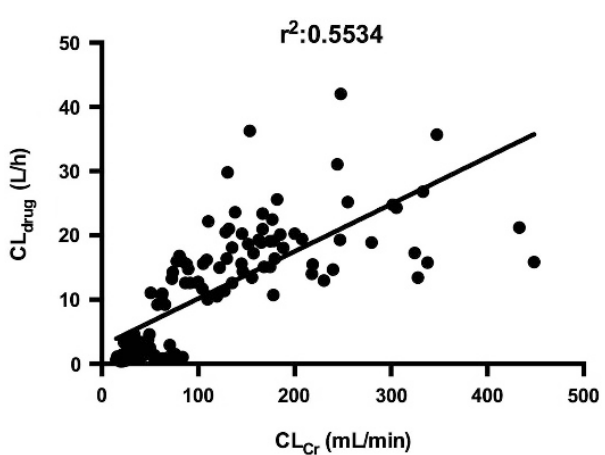

Figure 1 Linear correlation between creatinine clearance (CLCr) and total imipenem plasma clearance $\left(C L_{d r u g} ; r^{2}: 0.5534 ; P<0.0001\right)$.

In addition, PK data variability (CV\%) was estimated (means/s.d.) on the basis of stratified renal function as the prolongation of elimination half-life from $2.9 \pm 1.8 \mathrm{~h}(\mathrm{CV}: 63 \%)$ to $6.4 \pm 3.2 \mathrm{~h}$ (CV:50\%), reduction in plasma clearance from $17.8 \pm 6.41 \mathrm{~h}^{-1}(\mathrm{CV}$ : $36 \%)$ to $1.7 \pm 1.11 \mathrm{~h}^{-1}(\mathrm{CV}: 62 \%)$ and a reduced volume of distribution from $1.01 \pm 0.581 \mathrm{~kg}^{-1}(\mathrm{CV}: 58 \%)$ to $0.18 \pm 0.061 \mathrm{~kg}^{-1}$ (CV: 34\%) for NRF data sets compared with RF sets, respectively.

When burn patients with preserved renal function (NRF sets) presented renal dysfunction in a subsequent period during the clinical course (RF sets), the daily dose of imipenem was reduced to avoid the neurotoxicity of this carbapenem derivative. Drug plasma levels were monitored until renal function recovered to guarantee drug safety and efficacy in these intensive care unit patients. The prescribed daily dose of imipenem to burn patients in the NRF sets $\left(31.1 \mathrm{mg} \mathrm{kg}^{-1}\right)$ was reduced to $17.2 \mathrm{mg} \mathrm{kg}^{-1}$ to prevent drug toxicity (Table 2). Good correlation was obtained by plotting the creatinine clearance against imipenem plasma clearance $\left(r^{2}: 0.55\right)$ for NRF sets (Figure 1).

The PK parameters in patients with normal renal function and also with renal failure were also stratified according to the percentage of total burn surface area (TBSA) (Tables 3 and 4). Significant differences were obtained on PK parameters of imipenem according to TBSA in these patients with normal renal function, whereas no differences were observed on the daily dose and on the trough (Table 3 ).

On the other hand no significant differences were observed for the PK profile of imipenem according to TBSA changes in patients with renal failure, whereas to avoid neurotoxicity of imipenem in these patients, significant reduction was registered on the daily dose, and consequently on the trough (Table 4).

The PK/PD correlation for attained imipenem target doses was estimated according to $40 \% \mathrm{f} \mathrm{T}>\mathrm{MIC}$ considering renal function
(Figure 2). In addition, the percentage of PK/PD target achievement in burn patients who received antimicrobial therapy is described in Table 5.

\section{DISCUSSION}

Few data on the pharmacokinetics of imipenem ${ }^{6,7,10}$ and PK/PD correlations in burn patients are available. Physiological changes that occur after burns include decreased protein binding and increased glomerular filtration rate, which alters drug pharmacokinetics. Also, inter- and intra-patient variability in pharmacokinetics is a known characteristic of burn patients. ${ }^{2,3,6,7}$

The glomerular filtration rate falls during the acute phase of injury in burn patients. This effect lasts for $48 \mathrm{~h}$ and increases during the following hypermetabolic phase, which is also characterized by increased cardiac output. These changes directly influence the PK profile of imipenem by increasing drug plasma clearance as a consequence of tubular secretion and glomerular filtration rate in burn patients compared to non-burn or critically ill patients. $3,7,11,12$ Conflicting data on the pharmacokinetics of imipenem in burn patients have been reported. ${ }^{6,7}$ Boucher et al. ${ }^{6}$ reported PK data in burn patients with preserved renal function and renal failure of $\mathrm{CL}_{\mathrm{T}}$ : $12.5 \pm 3.61 \mathrm{~h}^{-1}, \mathrm{CV}: 29 \%$; $\mathrm{Vd}^{\mathrm{ss}}: 0.22 \pm 0.081 \mathrm{~kg}^{-1}, \mathrm{CV}: 36 \% ; \mathrm{t}_{(1 / 2)}$ $\beta: 1.12 \pm 0.44 \mathrm{~h}, \mathrm{CV}: 39 \%$, and Drusano et al. ${ }^{11}$ reported PK data of $\mathrm{CL}_{\mathrm{T}}: 12.1 \pm 0.061 \mathrm{~h}^{-1}, \mathrm{CV}: 0.5 \%$; Vdss: $0.26 \pm 0.051 \mathrm{~kg}^{-1}, \mathrm{CV}: 19 \%$; $t_{(1 / 2) \beta}: 0.93 \pm 0.09$ h, CV: $9.6 \%$ in healthy volunteers. ${ }^{6,11}$

However, Dailly et al. ${ }^{7}$ investigated 47 burn patients $48 \mathrm{~h}$ after burn injury and reported increased plasma clearance $\left(\mathrm{CL}_{\mathrm{T}}: 16.4 \pm 0.21 \mathrm{~h}^{-1}\right.$; CV: $1.3 \%)$ and volume of distribution $\left(0.38 \pm 0.04 \mathrm{l} \mathrm{kg}^{-1}, \mathrm{CV}: 10.5 \%\right)$ compared normal volunteers. ${ }^{7,11}$

In addition, Belzberg et al. ${ }^{12}$ reported an unpredictability of imipenem pharmacokinetics in critically ill patients (50 postsurgical patients) as a consequence of highly variable $\mathrm{PK}$ parameters $\left(\mathrm{CL}_{\mathrm{T}}: 12.1 \pm 12.0 \mathrm{lh}^{-1}, \mathrm{CV}: 99 \%\right.$; $\mathrm{Vd}^{\mathrm{ss}}: 0.57 \pm 0.481 \mathrm{~kg}^{-1}, \mathrm{CV}: 84 \%$; $\left.t_{(1 / 2) \beta}: 2.86 \pm 1.69 h, 59 \%\right)$. It is important to highlight that these critically ill patients had preserved renal function but several degrees of renal impairment. ${ }^{12}$

Data in the present study for imipenem kinetic disposition in burn patients with normal renal function (NRF sets) presented increases in plasma clearance $\left(17.8 \pm 6.41 \mathrm{~h}^{-1}\right.$; $\left.\mathrm{CV}: 36 \%\right)$, apparent volume of distribution $\left(1.01 \pm 0.581 \mathrm{~kg}^{-1}\right.$; CV: $\left.59 \%\right)$ and a prolonged biological half-life $(2.8 \pm 1.8 \mathrm{~h} ; \mathrm{CV}: 63 \%)$ compared with data in healthy volunteers and burn patients. ${ }^{6,11}$ In addition, PK data in the present study are consistent with Dailly et al. ${ }^{7}$ in burn patients and Bezberg et al. ${ }^{12}$ in critically ill non-burn patients. ${ }^{7,12}$

The obtained variability of kinetic parameters in the present study is consistent with data reported previously in non-burn critically ill 
Table 3 Data stratification for imipenem pharmacokinetic (PK) data based on total burn surface area for patient's NRF sets ( $n=73$ )

TBSA\%

\begin{tabular}{|c|c|c|c|c|}
\hline Parameter & $<19 \%(\mathrm{n}=14)$ & $20-39 \%(n=18)$ & $>40 \%(n=41)$ & P-value a \\
\hline$t_{(1 / 2) \beta}(h)$ & $1.5(1.3-2.1)$ & $2.0(1.5-2.6)$ & $3.2(1.8-4.8)$ & 0.0027 \\
\hline$C L_{T}\left(I h^{-1}\right)$ & $18.1(15.5-20.7)$ & $19.1(14.2-25.3)$ & $15.5(12.6-19.2)$ & 0.0431 \\
\hline$V_{d^{\mathrm{ds}}}\left(\mathrm{kg}^{-1}\right)$ & $0.56(0.47-0.85)$ & $0.84(0.61-1.19)$ & $0.97(0.70-1.52)$ & 0.0085 \\
\hline Daily dose $\left(\mathrm{mg} \mathrm{kg}^{-1}\right)$ & $27.6 \pm 2.8$ & $30.8 \pm 11.1$ & $32.4 \pm 10.4$ & 0.2901 \\
\hline Trough (mg $\left.\left.\right|^{-1}\right)$ & $1.50(1.05-3.35)$ & $1.80(1.40-2.70)$ & $2.70(1.50-4.90)$ & 0.1665 \\
\hline
\end{tabular}

$\mathrm{CL}_{T}$, total plasma clearance; NRF, normal renal function; $\mathrm{t}_{(1 / 2) \beta}$, biological half-life; TBSA, total burn surface area; $\mathrm{Vd}^{\mathrm{ss}}$, apparent volume of distribution at steady state.

aStatistics: one-way analysis of variance-Kruskal-Wallis test for PK and Tukey for daily dose. Data expressed as quartiles (median/lQ 25-75\%) for pharmacokinetics parameters and as mean \pm s.d. for daily dose and trough.

Table 4 Data stratification for imipenem pharmacokinetic data on the basis of total burn surface area for patient's RF sets ( $n=47$ )

\begin{tabular}{|c|c|c|c|c|}
\hline \multirow[b]{2}{*}{ Parameter } & \multicolumn{4}{|c|}{ TBSA\% } \\
\hline & $<19 \%(\mathrm{n}=19)$ & $20-39 \%(n=22)$ & $>40 \%(n=06)$ & P-value \\
\hline$t_{(1 / 2) \beta}(h)$ & $6.8(4.8-9.2)$ & $5.3(3.9-7.4)$ & $6.0(4.8-10.6)$ & 0.4688 \\
\hline $\mathrm{CL}_{T}\left(\mathrm{I} \mathrm{h}^{-1}\right)$ & $1.1(0.7-2.0)$ & $1.6(1.1-2.6)$ & $1.4(1.0-1.7)$ & 0.2232 \\
\hline$V d^{s s}\left(\mid k g^{-1}\right)$ & $0.21(0.13-0.23)$ & $0.19(0.15-0.22)$ & $0.19(0.14-0.24)$ & 0.9890 \\
\hline Daily dose $\left(\mathrm{mg} \mathrm{kg}^{-1}\right)$ & $12.5 \pm 8.3$ & $20.7 \pm 10.2$ & $18.8 \pm 7.2$ & 0.0198 \\
\hline Trough $\left(\mathrm{mgl}^{-1}\right)$ & $6.8(4.8-9.2)$ & $8.2(5.8-11.0)$ & $3.8(3.3-6.1)$ & 0.0270 \\
\hline
\end{tabular}

Abbreviations: $\mathrm{CL}_{\mathrm{T}}$, total plasma clearance; NRF, normal renal function; $\mathrm{t}_{(1 / 2) \beta}$, biological half-life; TBSA, total burn surface area; Vdss, apparent volume of distribution at steady state.

aStatistics: one-way analysis of variance-Kruskal-Wallis test for PK and Tukey for daily dose. Data expressed as quartiles (median/IQ 25-75\%) for pharmacokinetics parameters and as mean \pm s.d. for daily dose and trough.

a

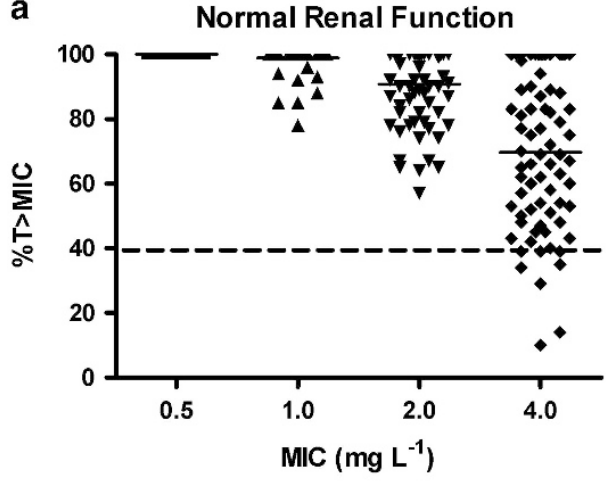

b

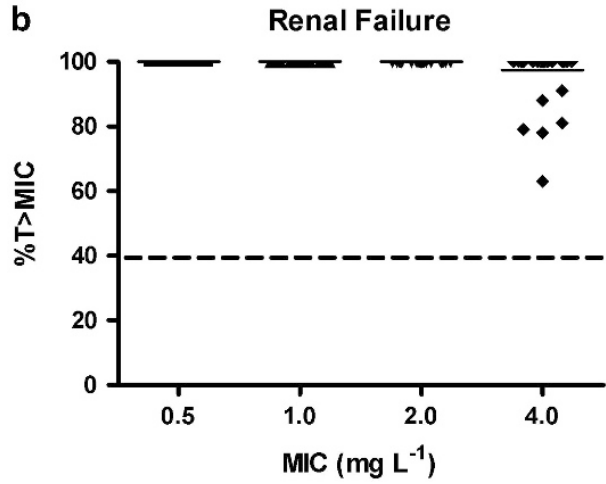

Figure 2 Probability of target attainment of imipenem for two patient populations for a range of MICs. (a) Normal renal function (73 sets) (b) renal failure (47 sets). PK/PD correlation for MIC values from $0.5 \mathrm{mgl}^{-1}$ to $4.0 \mathrm{mgl}^{-1}$. MIC, minimum inhibitory concentration.
Table 5 Probability of target attainment in burn patients submitted to imipenem antimicrobial therapy

\begin{tabular}{lcccc}
\hline & \multicolumn{4}{c}{$\left.M I C(m g)^{-1}\right)$} \\
\cline { 2 - 5 } & 0.5 & 1 & 2 & 4 \\
\hline Imipenem (40\%fT>MIC) & & & & \\
$\quad$ Normal renal function $(n=73)$ & 100.0 & 100.0 & 100.0 & 89.0 \\
Renal failure $(n=47)$ & 100.0 & 100.0 & 100.0 & 100.0
\end{tabular}

Abbreviations:MIC, minimum inhibitory concentration; PK/PD, pharmacokinetic/ pharmacodynamics.

PK/PD correlation: $(40 \% f \mathrm{~T}>\mathrm{MIC})$ : imipenem index of effectiveness based on period of time dosing interval that drug plasma concentration remains above the MIC.

patients. These findings could be justified on several factors that alter the pharmacokinetics of imipenem in critically ill burn patients and non-burns patients from the intensive care unit compared with healthy volunteers. ${ }^{7,11,12}$

The present study demonstrated significant differences in the biological half-life of imipenem in burn patients with normal renal function as a consequence of increases in the volume of distribution according to the increases in TBSA $>40 \%$.

In addition, no changes related to imipenem PK profile according to TBSA were observed. In contrast to the logically expected outcome, no difference in imipenem pharmacokinetics related to TBSA was obtained, as reported previously. ${ }^{6}$

These findings may explain the antimicrobial effective drug plasma concentration range because no leakage through the burn wound is expected compared with vancomycin. 
The usefulness of the correlation between drug plasma clearance and creatinine clearance relates to the ability of designing dose regimens to achieve the desired therapeutic goals. Several pharmacokinetic investigations have demonstrated a good relationship between creatinine clearance and total plasma clearance for drugs with a significant renal route of elimination in burn patients. A good correlation between drug plasma clearance and creatinine clearance was demonstrated for imipenem in the present study, which is consistent with previous published data. ${ }^{6,7}$

One constant concern is bacterial resistance and a reduced development of new agents. Therefore, it is imperative to optimize antimicrobial exposures for available agents via the maximization of patient outcome and the minimization of the potential for further resistance. Pharmacokinetics could be an important tool for antimicrobial efficacy via the prediction of drug exposure, but pharmacodynamic goals and pathogen susceptibility must also be considered to ensure clinical efficacy. Several PK/PD indices have recently emerged as surrogate markers for successful clinical outcomes of antimicrobial therapy. These indices combine patient-specific PK parameters on the basis of drug plasma levels with a specific susceptibility data about the pathogen in question. Microbial susceptibility to the antimicrobial agent (MIC values) for the isolated pathogen from fluids and secretion obtained from the patient can be determined in a central laboratory at the hospital. In addition, the associated MIC variability can be obtained within a typical clinical setting or surveillance databases, such as EUCAST. ${ }^{13}$ PK/PD correlation is an important tool to predict dose efficacy, and it is an important goal for antimicrobial monitoring. $4,10,13$

In the present study, a high percentage of target attainment was shown for imipenem using the PK/PD correlation (above $89 \%$ for all MICs: 0.5 to $4.0 \mathrm{mgl}^{-1}$ ). Contrary to expectations, no dose adjustments for imipenem were required for patients with normal renal function. However, the daily dose must be reduced according to the patient's renal insufficiency to avoid neurotoxicity.

This study included the following limitations: (i) determination of drug total body clearance by calculation rather than direct measurements from blood and urine collection; (ii) the use of the estimated creatinine clearance as determined by the Cockcroft-Gault equation as a measure of a patient's renal function, considering different normalization on the basis of gender as recommended; ${ }^{6}$ (iii) assumptions that weight on admission was reflective of the patient's weight throughout their entire stay in the ICBU; and (iv) MIC values from surveillance databases instead of the clinical setting was considered if the pathogen in fluids or secretions obtained for a patient could not be isolated in the Central Laboratory in any data set during the follow-up period in the ICBU.

The high variability of imipenem kinetic disposition in burn patients emphasized the importance of imipenem plasma monitoring during antimicrobial therapy in burn patients to guarantee drug efficacy and safety.

Therefore, the PK/PD correlation should be conducted periodically as part of routine procedures performed in the ICBU during antimicrobial therapy, even after target achievement in all patients.

Finally, the pharmacokinetics of imipenem is altered in burn patients in the ICBU during the hypermetabolic phase, and renal function influenced PK parameters.

In conclusion, a high probability of imipenem target attainment (MICs $0.5-4.0 \mathrm{mg} \mathrm{l}^{-1}$ ) related to drug efficacy was obtained via the application of the $\mathrm{PK} / \mathrm{PD}$ correlation, but daily doses required reductions in patients with renal failure to avoid neurotoxicity.

\section{CONFLICT OF INTEREST}

The authors declare no conflict of interest.

\section{ACKNOWLEDGEMENTS}

Brazilian Foundation for Research, CAPES and FAPESP.

Author contributions: All the authors were involved in the study conception and design, data acquisition or data analysis and interpretation. DSG, CS-G and SRCJS were involved in drafting or revising the article critically for important intellectual content and final approval of the submitted version.

1 Fry, D. E. The importance of antibiotic pharmacokinetics in critical illness. Am. J. Surg. 172, 20S-25S (1996).

2 Weinbren, M. J. Pharmacokinetics of antibiotics in burn patients. J. Antimicrob. Chemother. 44, 319-327 (1999).

3 Blanchet, B., Jullien, V., Vinsonneau, C., Tod, M. Influence of burns on pharmacokinetics and pharmacodynamics of drugs used in the care of burn patients. Clin. Pharmacokinet. 47, 635-654 (2008).

4 Scaglione, F. Pharmacokinetic/pharmacodynamic (PK/PD) considerations in the management of Gram-positive bacteremia. Int. J. Antimicrob. Agents 36S, S33-S39 (2010).

5 Varghese, J. M., Roberts, J. A., Lipman, J. Antimicrobial pharmacokinetic and pharmacodynamic issues in the critically ill with severe sepsis and septic shock. Crit. Care Clin. 27, 19-34 (2011).

6 Boucher, B. A., Hickerson, W. L., Kuhl, D. A., Bombassaro, A. M., Jaresko, G. S. Imipenem pharmacokinetics in patients with burns. Clin. Pharmacol. Ther. 48, 130-137 (1990)

7 Dailly, E., Kergueris, M. F., Pannier, M., Jolliet, P., Bourin, M. Population pharmacokinetics of imipenem in burn patients. Fundam. Clin. Pharmacol. 17, 645-650 (2003).

8 Cockcroft, D. W., Gault, M. H. Prediction of creatinine clearance from serum creatinine. Nephron 16, 31-41 (1976).

9 European Committee of Antimicrobial Susceptibility Testing. MIC distribution, available on www.eucast.org/mic distributions/ accessed 2013-2014.

10 Ikawa, K., Morikawa, N., Ohge, K. I. H., Sueda, T. Development of breakpoints of carbapenems for intra-abdominal infections based on pharmacokinetics and pharmacodynamics in peritoneal fluid. J. Infect. Chemother. 14, 330-332 (2008).

11 Drusano, G. L. et al. Multiple-dose pharmacokinetics of imipenem-cilastatin. Antimicrob. Agents Chemother. 26, 715-721 (1984).

12 Belzberg, $H$. et al. Imipenem levels are not predictable in the critically ill patient. J. Trauma 56, 111-117 (2004).

13 Schmidt, S., Barbour, A., Sahre, M., Rand, K. H., Derendorf, H. PK/PD: new insights for antibacterial and antiviral applications. Curr. Opin. Pharmacol. 8, 549-556 (2008). 